ACCEPTED MANUSCRIPT

\title{
Effective interactions in twisted double-layer graphene in a microcavity
}

To cite this article before publication: Juan Sebastian Ardenghi $2020 \mathrm{~J}$. Phys.: Condens. Matter in press https://doi.org/10.1088/1361-648X/ab8aff

\section{Manuscript version: Accepted Manuscript}

Accepted Manuscript is "the version of the article accepted for publication including all changes made as a result of the peer review process, and which may also include the addition to the article by IOP Publishing of a header, an article ID, a cover sheet and/or an 'Accepted Manuscript' watermark, but excluding any other editing, typesetting or other changes made by IOP Publishing and/or its licensors"

This Accepted Manuscript is @ 2020 IOP Publishing Ltd.

During the embargo period (the 12 month period from the publication of the Version of Record of this article), the Accepted Manuscript is fully protected by copyright and cannot be reused or reposted elsewhere.

As the Version of Record of this article is going to be / has been published on a subscription basis, this Accepted Manuscript is available for reuse under a CC BY-NC-ND 3.0 licence after the 12 month embargo period.

After the embargo period, everyone is permitted to use copy and redistribute this article for non-commercial purposes only, provided that they adhere to all the terms of the licence https://creativecommons.org/licences/by-nc-nd/3.0

Although reasonable endeavours have been taken to obtain all necessary permissions from third parties to include their copyrighted content within this article, their full citation and copyright line may not be present in this Accepted Manuscript version. Before using any content from this article, please refer to the Version of Record on IOPscience once published for full citation and copyright details, as permissions will likely be required. All third party content is fully copyright protected, unless specifically stated otherwise in the figure caption in the Version of Record.

View the article online for updates and enhancements. 


\section{Introduction}

Graphene, a monolayer of carbon atoms have aroused enormous interest due to its potential applications ([1], [54] and [3]). Recently, double-layer graphene (DLG) has acquire considerable interest due to appearance of exotic quantum states like fractional quantum Hall effect due to the interactions of electrons both whitin and across graphene layers ([4]). In turn, the possibility of excitonic condensation in DLG with and without negligible hopping between graphene sheets has been studied carefully in ([5] and [6]), where the excitonic gap can reach values on the order of the Fermi energy at strong interactions, taking into account the screening of the interlayer Coulomb interaction by the carriers in the layers. For ABC stacked few-layer graphene, strongly correlated electron-hole pairing regime is accesible to observe superfluidity at enhanced densities [7]. Rotational stacking faults in multilayer graphene dramatically reduce interlayer coherence and increase the magnitudes of correlation energies and decrease quasiparticle velocities [8]. Moreover, a magic angle has been found for bilayer graphene twist at which the stack seems to exhibit superconductivity ([9], [10] and [11]) . The favourable conditions for these excitonics effects can be achieved when $k_{F} d<1$ where $d$ is the distance between layers. In this limit, the behavior of the system is determined by the dimensionless parameter $r_{s}=$ $\langle V\rangle\left\langle E_{F}\right.$ that for graphene is $r_{s}=e^{2} / \kappa \hbar v_{F}$ and is independent of the carrier density $n$. In general, when two graphene layers are present, correlations between them should appears due to the strong coupling of electron-electron interactions even when they cannot exchange particles, provided that the layer separation $d$ is comparable to a characteristic distance $l$ between charge carriers within layers [12]. One of the consequences of this remote coupling is a phenomenon called Coulomb drag, in which

*email: jsardenghi@gmail.com, fax number: +54-291-4595142 
an electric current passed through one of the layers causes frictional charge flow in the other layer, even when both layers are neutral [13]. In turn, entanglement generation in double-layer graphene in a microcavity is possible due to the vacuum fluctuations of the electromagnetic field cavity [14].

On the other side, the composite electron-photon states, known as polariton excitations exhibit a bandgap when a graphene sheet is illuminated by circularly polarized light [15]. In general, the electron-quantum field interaction is considered within the regime of weak light-matter coupling, where the interaction results only in electron transitions between unperturbed electron states, which are accompanied by absorption and emission of photons. However, the regime of strong light-matter interactions cannot be described as a weak perturbation. In this case, the system consists of a bound electron-photon system, which was called "electron dressed by photons" and became a commonly used model in modern physics ([17] and [18]). In this strong regime, a lot of interesting physical effects can be expected, such as optical planar microcavities with semiconductor $(\{19]$ and $[20])$, microcavities with individual quantum dots ([21] and [22]) and others. Effects of strong coupling can be used for variety of technological applications [23], including novel types of the lasers ([24] and [25]), optical switches and logic gates ([26] and [27]), sources of entangled photon pairs [28] and others. In graphene related research, the attention has been paid to the field-induced modification of energy spectrum of dressed electrons ([29], [30], [31], [15], [32] and [33]), optical response of dressed electrons [34], transport of dressed electrons in graphene-based p-n junctions [35] and electronic transport through dressed edge states in graphene ([36], [37] and [38]).

Combining the effects of light-matter coupling in a microcavity with the unusual properties of twisted double-layer graphene, in this work the effective interactions between electrons in different layers are studied by applying the Schrieffer-Wolff transformation (SW) with the condition $k_{F} d>1$ and negligible carrier density $n$ in each layer. These conditions do not allow excitonic effects and in turn, the intralayer Coulomb interaction can be neglected. The SW has been used extensively in different areas of physics under different names [39] and can be thought of as a kind of renormalization procedure because not only diagonalizes the Hamiltonian in a perturbative manner, but it also renormalizes the parameters in the free Hamiltonian ([40], [41]). Based on a canonical transformation of the Hamiltonian $e^{S} H^{-S}$, with an appropiate choice of $S$, the transformed Hamiltonian leads to a low-energy effective Hamiltonian that contains interactions between carriers in different layers of graphene due to the quantum correlations originated from each layer with the electromagnetic field in the microcavity. In this work, we show that this correlation appears as a type of pseudospin exchange $\sim-J_{D P}\left[\cos \theta \sigma_{\mathbf{1}} \cdot \sigma_{\mathbf{2}}+\sin \theta\left(\sigma_{1} \times \sigma_{\mathbf{2}}\right) \cdot \widehat{e}_{z}\right]$ where the coupling constant depends strongly in the layers height, the photon energy and the rotation angle $\theta$ between layers. Manipulating this effective interaction can be performed by an independent control over the carrier type and density on each layer, easily realized with gate voltages. The manuscript will be organized as follows: In Section II, the Schrieffer-Wolff transformation is applied and the operator $S$ is found. In section III, the low-energy effective interaction is obtained where the photon subspace is projected out and is analyzed. In last section, the conclusions are presented.

\section{The Schrieffer-Wolf transformation}

Consider two copies of graphene, one on the top of another, forming a double-layer graphene, where interlayer transitions are neglected (see figure $1 \mathrm{a}$ )). This approximation can be applied when both graphene layers are separated a distance $\Delta d=\left|d_{1}-d_{2}\right|$ much larger than the mean distance between electrons in each layer given by $\left\langle r_{0}\right\rangle=1 / \sqrt{\pi n}$, where $n$ is the carrier density. In graphene $k_{F}=\sqrt{\pi n}$, then near the Dirac point $k_{F} \sim 0$ and $n \sim 0$ and $\left\langle r_{0}\right\rangle \rightarrow \infty$, which implies that intralayer-Coulomb interaction is negligible. In turn, if $k_{F} \Delta d>1$ then $\left.\Delta d\right\rangle\left\langle r_{0}\right\rangle$ which implies that the interlayer Coulomb interaction $\sim \Delta d^{-1}$ can be neglected. Then by tuning $n$ with a gate voltage near the Dirac point, the Hamiltonian of two copies of graphene without interaction between them is a good approximation for negligible carrier density and large interlayer separation. Hopping energy between layers can be neglected for $\Delta d>3 \AA$ which is the typical distance between layers in graphite and larger than the 


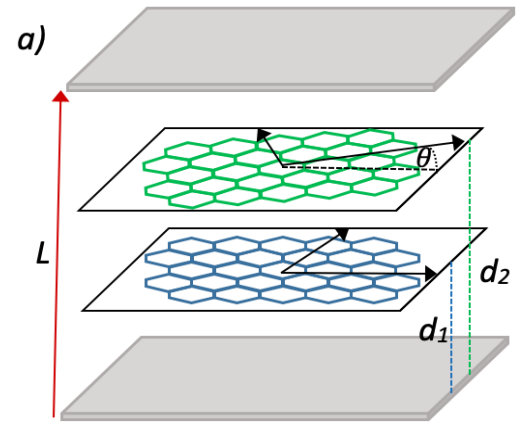

b)

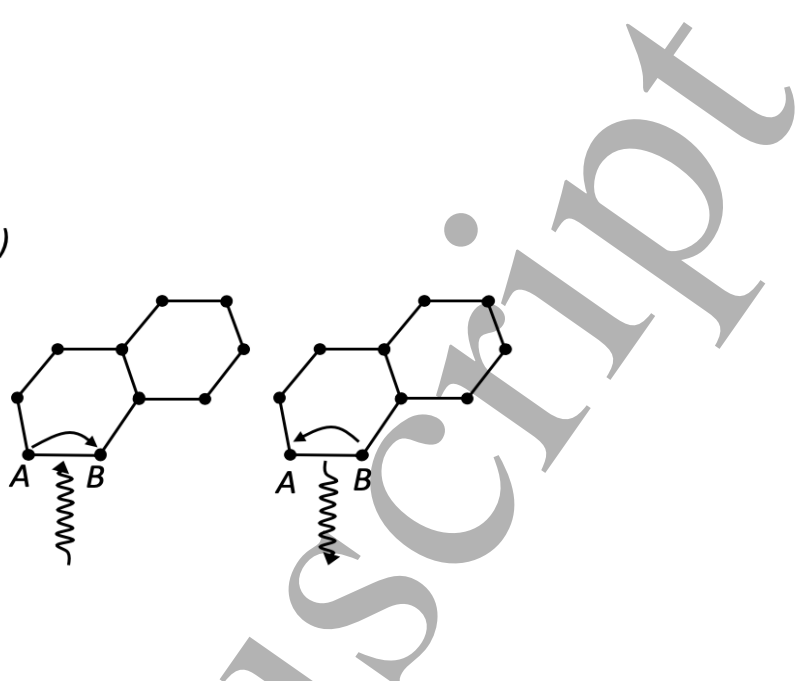

Figure 1: a) Microcavity setup with twisted double-layer graphene. b) Diagrammatic description of Hamiltonian of eq.(3). Emission and absorption of one photon with energy hw and electron sublattice transition

interaction parameter $r_{s}<2.2$ (see [54]). Now we can proceed with a twist of one layer an angle $\theta / 2$ and the other layer an angle $-\theta / 2$, then the total angle twist is $\theta$ (see figure 1a)). By rotating the Pauli matrices the twisted Hamiltonian of the decoupled graphene layers read (see [42] $)^{1}$

$$
H_{0 T}=\sum_{i=1,2} v_{F}\left(e^{-i \theta_{i}} \sigma_{-}^{(i)} p_{-}^{(i)}+e^{i \theta_{i}} \sigma_{+}^{(i)} p_{+}^{(i)}\right)
$$

where $\sigma_{ \pm}^{(i)}=\frac{1}{2}\left(\sigma_{x}^{(i)} \pm i \sigma_{y}^{(i)}\right)$ and $p_{ \pm}^{(i)}=\frac{1}{2}\left(p_{x}^{(i)} \pm i p_{y}^{(i)}\right)$ and $\theta_{1}=\theta / 2$ and $\theta_{2}=-\theta / 2$. If we introduce the double-layer graphene in a microcavity of length $L$ with a monochromatic electromagnetic wave, both layers are coupled to this electromagnetic field via the minimum coupling $\mathbf{p} \rightarrow \mathbf{p}+\frac{e}{c} \mathbf{A}$, where $\mathbf{A}$ is the potential vector (see eq.(1) of [31]). This implies that a dot product between $\sigma$ and $\mathbf{A}$ appears (similar to $\sigma$ and $\mathbf{p}$ ) an identical result is obtained with $p_{ \pm}^{(i)}$ replaced by $A_{ \pm}^{(i)}=\frac{1}{2}\left(A_{x}^{(i)} \pm i A_{y}^{(i)}\right)$ in last equation (see eq.(4) of [31]).Considering/a clockwise polarized electromagnetic wave with wave frequency $\omega$ propagating perpendicular to the graphene layers, the potential vector acting on each layer can be written as (see eq. (2) of $[15])^{2}$

$$
\mathbf{A}_{i}=\sqrt{\frac{2 \pi \hbar c^{2}}{\epsilon \omega V}} \sin \left(\frac{m \pi d_{i}}{L}\right)\left(\widehat{e}_{+}^{(i)} a+\widehat{e}_{-}^{(i)} a^{\dagger}\right)
$$

where $\widehat{e}_{\alpha}^{(j)}=\frac{1}{2}\left(\widehat{e}_{x}^{(j)}+i \alpha \widehat{e}_{y}^{(j)}\right)$ are the polarization vectors, $\widehat{e}_{x, y}^{(j)}$ are the unit vectors directed along the $x$ and $y$ axis, $V=S L$ is the volume of the cavity, where $S$ is the area of the graphene sheet and $\epsilon$ is the dielectric constant and $a$ and $a^{\dagger}$ are creation(annihilation) operators of the cavity mode of the photon field with frequency $\omega=\frac{c}{n_{0}} \frac{m \pi}{L}$, where $m \in \mathbb{N}, n_{0}$ is the refractive index of the dielectric in which the circular polarized light travels in the microcavity and $c$ is the velocity of light in the vacuum. The $a$ and $a^{\dagger}$ operators obeys the usual commutation relations $\left[a, a^{\dagger}\right]=I$. Should be stressed that in the case of real microcavities, in general, the reflectivity of the mirrors is finite, then a standing wave mode of the cavity decays. This is a dissipation process arisen from nonideal structure of the cavity and is usually parametrized by the $Q$-factor of the resonator and lead to the finite spectral linewidth of the cavity mode. For simplicity we will ignore cavity decays which implies that the cavity mode is not broaden. The interaction Hamiltonian between twisted double-layer graphene and the potential vector $-e v_{F} \sigma^{(i)} \cdot \mathbf{A}^{(i)}$ reads

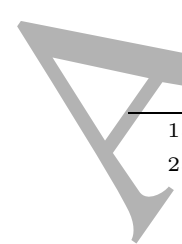

$$
H_{\text {int }}=-\sum_{i=1,2} \gamma_{i}\left(e^{-i \theta_{i}} \sigma_{-}^{(i)} a+e^{i \theta_{i}} \sigma_{+}^{(i)} a^{\dagger}\right)
$$

${ }^{1}$ The rotation implies that $\sigma_{x}(\theta)=e^{-i \theta} \sigma_{-}+e^{i \theta} \sigma_{+}$and $\sigma_{y}(\theta)=i e^{i \theta} \sigma_{+}-i e^{-i \theta} \sigma_{-}$, where $\sigma_{ \pm}=\frac{1}{2}\left(\sigma_{x} \pm i \sigma_{y}\right)$.

${ }^{2}$ The general expression for the potential vector for both polarizations can be seen in [31]. 
where $\gamma_{i}=\sin \left(\frac{m \pi d_{i}}{L}\right)$. Finally, adding the electromagnetic field Hamiltonian $H_{0 F}=\hbar \omega a^{\dagger} a$, we can write

$$
H=H_{0 T}+H_{0 F}+g H_{i n t}
$$

where $g=e \sqrt{\frac{2 \pi \hbar v_{F}^{2}}{\epsilon \omega_{m} V}}$ is the coupling constant ${ }^{3}$. Hamiltonian of eq. (3) contains two processes: an electron jumps from sublattice $A$ to $B$ by absorbing a photon with energy $\hbar \omega$ and the inverse process, where an electron jumps from $B$ to $A$ by emmiting a photon with energy $\hbar \omega$ (see figure $1 \mathrm{~b}$ ). In order to obtain the effective interaction between the graphene layers and the electromagnetic field, we can apply the Schrieffer-Wolff transformation ([39],[40]) or Löwdin partitioning [43]. This transformation is similar to the conventional stationary perturbation theory but with the improve that does not distinguish between degenerate and non-degenerate states. By considering a unitary anti-Hermitian operator $S$ and an unitary transformation $e^{g S} H e^{-g S}$ on $H$, the transformed Hamiltonian must have a block-diagonal form. The only requirement for the Hamiltonian $H$ is that the matrix elements of the interaction $H_{\text {int }}$ are small with respect to the eigenvalues of the matrix $H_{0 F}$, then by using the Campbell-Hausdorff expansion, $H^{\prime}$ can be expresssed as

$$
H^{\prime}=e^{g S} H e^{-g S}=H+g[S, H]+\frac{g^{2}}{2}\left[S,[S, H], 4 O\left(g^{3}\right)\right.
$$

by replacing $H=H_{0 S}+H_{0 F}+g H_{\text {int }}$ in last equation and imposing that linear order in $g$ in $H^{\prime}$ vanishes, we obtain an equation for $S$ such that $H_{\text {int }}+\left[S, H_{0 S}+H_{0 F}\right]=0$. Then last equation reads

$$
H^{\prime}=e^{g S} H e^{-g S}=H_{0 S}+H_{0 F}+\frac{g^{2}}{2}\left[S, H_{i n t}\right]+O\left(g^{3}\right)
$$

The transformed Hamiltonian $H^{\prime}$ contains interactions at order $g^{2}$. In order to obtain the matrix operator $S$ we can consider the following ansatz

$$
S=\sum_{i, \alpha} \sigma_{\alpha}^{(i)}\left(X_{\alpha+}^{(i)} a^{\dagger}-X_{\alpha-}^{(i)} a\right)+\sum_{i} \sigma_{z}^{(i)}\left(W_{+}^{(i)} a^{\dagger}-W_{-}^{(i)} a\right)
$$

where the twelve unknown coefficients $X_{\alpha \alpha^{\prime}}^{(i)}$ and $W_{\alpha}^{(i)}$ must be determined through the equation $H_{\text {int }}+$ $\left[S, H_{0 S}+H_{0 F}\right]=0$. Using that $\left[\sigma_{z}^{(i)}, \sigma_{\alpha}^{(j)}\right]=2 \alpha \delta_{i j} \sigma_{\alpha}^{(i)}$ and $\left[\sigma_{\alpha}^{(i)}, \sigma_{\alpha^{\prime}}^{(j)}\right]=\alpha \delta_{i j} \delta_{\alpha,-\alpha^{\prime}} \sigma_{z}^{(i)}$ for the Pauli matrices and $\left[a^{\dagger}, a^{\dagger} a\right]=-a^{\dagger}$ and $\left[a, a^{\dagger} a\right]=a$ for the creation and annihilation operators, through a lengthly but straightforward calculation we obtain the following set of equations for the twelve unknown coefficients $X_{\alpha \alpha^{\prime}}^{(i)}$ and $W_{\alpha}^{(i)}$ condensate in three equations

$$
\begin{gathered}
\gamma_{i} e^{-i \alpha \theta_{i}}=-\hbar \omega X_{-\alpha, \alpha}^{(i)}-2 v_{F} e^{-i \alpha \theta_{i}} p_{\alpha}^{(i)} W_{\alpha}^{(i)} \\
2 v_{F} e^{i \alpha \theta_{i}} p_{-\alpha}^{(i)} W_{\alpha}^{(i)}-\hbar \omega X_{\alpha, \alpha}^{(i)}=0 \\
v_{F} e^{-i \alpha \theta_{i}} p_{\alpha}^{(i)} X_{\alpha, \alpha}^{(i)}-v_{F} e^{i \alpha \theta_{i}} p_{-\alpha}^{(i)} X_{-\alpha, \alpha}^{(i)}-\hbar \omega W_{\alpha}^{(i)}=0
\end{gathered}
$$

where $\alpha= \pm 1$ and $i=1,2$. Last system of equations can be solved with the following results

$$
\begin{gathered}
X_{\alpha, \alpha}^{(i)}=\frac{2 \gamma_{i} e^{i \alpha \theta_{i}}}{\hbar \omega} \frac{v_{F}^{2} p_{i}^{2} e^{-2 i \alpha \varphi_{i}}}{\hbar^{2} \omega^{2}-4 v_{F}^{2} p_{i}^{2}} \\
X_{-\alpha, \alpha}^{(i)}=\frac{\gamma_{i}}{\hbar \omega} e^{-i \alpha \theta_{i}} \frac{2 v_{F}^{2} p_{i}^{2}-\hbar^{2} \omega^{2}}{\hbar^{2} \omega^{2}-4 v_{F}^{2} p_{i}^{2}} \\
W_{\alpha}^{(i)}=\frac{\gamma_{i} v_{F} p_{i} e^{-i \alpha \varphi_{i}}}{\hbar^{2} \omega^{2}-4 v_{F}^{2} p_{i}^{2}}
\end{gathered}
$$

${ }^{3}$ Hamiltonian of eq.(3) resembles the Jaynes-Cumming model [16]. 
where $p_{i}=\sqrt{p_{x_{i}}^{2}+p_{y_{i}}^{2}}=\sqrt{p_{+}^{(i)} p_{-}^{(i)}}$ and $\varphi_{i}=\arctan \left(p_{y_{i}} / p_{x_{i}}\right)$ is the angle in the momentum space of each layer. With the knowledge of the anti-Hermitian operator $S$, we can compute the second order contribution in $g$ to $H$ by computing the commutator $\left[S, H_{\text {int }}\right]$. By using that $\left[\sigma_{z}^{(i)} a, \sigma_{\alpha}^{(j)} a^{\dagger}\right]=\sigma_{z}^{(i)} \sigma_{\alpha}^{(j)}+$ $2 \alpha \delta_{i j} \sigma_{\alpha}^{(i)} a^{\dagger} a,\left[\sigma_{z}^{(i)} a^{\dagger}, \sigma_{\alpha}^{(j)} a\right]=-\sigma_{z}^{(i)} \sigma_{\alpha}^{(j)}+2 \alpha \delta_{i j} \sigma_{\alpha}^{(i)} a a^{\dagger},\left[\sigma_{\alpha}^{(i)} a^{\dagger}, \sigma_{\alpha^{\prime}}^{(j)} a\right]=-\sigma_{\alpha}^{(i)} \sigma_{\alpha^{\prime}}^{(j)}+\alpha \delta_{i j} \delta_{\alpha,-\alpha^{\prime}} \sigma_{z}^{(i)} a a^{\dagger}$ and $\left[\sigma_{\alpha}^{(i)} a, \sigma_{\alpha^{\prime}}^{(j)} a^{\dagger}\right]=\sigma_{\alpha}^{(i)} \sigma_{\alpha^{\prime}}^{(j)}+\alpha \delta_{i j} \delta_{\alpha,-\alpha^{\prime}} \sigma_{z}^{(i)} a^{\dagger} a$ it can be shown that the contribution to second order in $g$ to the effective Hamiltonian reads

$$
\begin{gathered}
\left.\left[S, H_{i n t}\right]=\sum_{i, j, \alpha} \gamma_{j} e^{i \alpha \theta_{j}} W_{\alpha}^{(i)} \sigma_{z}^{(i)} \sigma_{\alpha}^{(j)}+\sum_{i, j, \alpha, \alpha^{\prime}} \gamma_{j} e^{i \alpha^{\prime} \theta_{j}} X_{\alpha \alpha^{\prime}}^{(i)} \sigma_{\alpha}^{(i)} \sigma_{\alpha^{\prime}}^{(j)}\right) \\
+\sum_{i} \gamma_{i} e^{-i \theta_{i}}\left(2 W_{+}^{(i)} \sigma_{-}^{(i)}-X_{++}^{(i)} \sigma_{z}^{(i)}\right) a^{\dagger} a^{\dagger}+\sum_{i} \gamma_{i} e^{i \theta_{i}}\left(2 W_{-}^{(i)} \sigma_{+}^{(i)}-X_{-}^{(i)} \sigma_{z}^{(i)}\right) a a+ \\
+\sum_{i, \alpha} \gamma_{i} e^{i \theta_{i}}\left(X_{-+}^{(i)} \sigma_{z}^{(i)}-2 W_{+}^{(i)} \sigma_{+}^{(i)}\right) a a^{\dagger}+\sum_{i, \alpha} \gamma_{i} e^{-i \theta_{i}}\left(X_{+-}^{(i)} \sigma_{z}^{(i)}-2 W_{-}^{(i)} \sigma_{-}^{(i)}\right) a^{\dagger} a
\end{gathered}
$$

The last four terms of last equation contain photon operators for emission and absorbtion of two photons and corrections to the photon field energy. This correction gives the AC-Stark shift. The first and second contribution introduce corrections to the $H_{0 S}$ term in the Hamiltonian and contain the effective interaction between the electrons in different graphene layers. This effective interaction between layers can be rooted to the absorption-emission photon process when electrons in different layers change sublattices. A second order transition implies that one electron emits a photon with a sublattice transition $A \rightarrow B$ in one layer and an electron in the other layer absorbs the same photon with a transition $B \rightarrow A$. In this case, the photon is virtual and cannot be observed, although renormalizes the bare parameters in the initial Hamiltonian.

\section{Effective interactions of twisted double-layer graphene}

In order to study the effective interactions that appears between electrons in different graphene layers, we can restrict us to the first two terms of eq.(10)

$$
\left[S, H_{i n t}\right]_{e l}=H_{e f}^{\prime}+H_{e f}^{\prime \prime}
$$

where

and

$$
H_{e f}^{\prime}=\sum_{i, \alpha} \gamma_{i} e^{i \alpha \theta_{i}} W_{\alpha}^{(i)} \alpha \sigma_{\alpha}^{(j)}+\sum_{i, \alpha \neq \alpha^{\prime}} \gamma_{i} e^{i \alpha^{\prime} \theta_{i}} X_{\alpha \alpha^{\prime}}^{(i)} \sigma_{\alpha}^{(i)} \sigma_{\alpha^{\prime}}^{(i)}
$$

$$
H_{e f}^{\prime \prime}=\sum_{i \neq j, \alpha} \gamma_{j} e^{i \alpha \theta_{j}} W_{\alpha}^{(i)} \sigma_{z}^{(i)} \sigma_{\alpha}^{(j)}+\sum_{i \neq j, \alpha, \alpha^{\prime}} \gamma_{j} e^{i \alpha^{\prime} \theta_{j}} X_{\alpha \alpha^{\prime}}^{(i)} \sigma_{\alpha}^{(i)} \sigma_{\alpha^{\prime}}^{(j)}
$$

where we have used that $\sigma_{z}^{(i)} \sigma_{\alpha}^{(i)}=\alpha \sigma_{\alpha}^{(i)}$ and $\sigma_{\alpha}^{(i)} \sigma_{\alpha}^{(i)}=0$. Basically, we have considered the first two terms in eq.(10) and we have written $\sum_{i, j}=\sum_{i=j}+\sum_{i \neq j}$ in eq.(10), then the first Hamiltonian $H_{e f}^{\prime}$ contains only one body potentials terms that do not couple the layers and the crossed terms between layers appear only in $H_{e f}^{\prime \prime}$. The second sum in $H_{e f}^{\prime}$ can be written as

$$
\sum_{i, \alpha \neq \alpha^{\prime}} \gamma_{i} e^{i \alpha^{\prime} \theta_{i}} X_{\alpha \alpha^{\prime}}^{(i)} \sigma_{\alpha}^{(i)} \sigma_{\alpha^{\prime}}^{(j)}=\sum_{i}\left(\frac{\gamma_{i}^{2}}{\hbar \omega} \frac{2 v_{F}^{2} p_{i}^{2}-\hbar^{2} \omega^{2}}{\hbar^{2} \omega^{2}-4 v_{F}^{2} p_{i}^{2}}\right) I_{i}
$$

where we have used that $\sigma_{\alpha}^{(i)} \sigma_{-\alpha}^{(i)}=\frac{I_{i}+\alpha \sigma_{z}^{(i)}}{2}, X_{-,+}^{(i)} e^{i \theta_{i}}=X_{+,-}^{(i)} e^{-i \theta_{i}}$ and the obtained value of $X_{+,-}^{(i)}$ in eq. (9). The last term introduces different diagonal matrix elements in the independent Hamiltonians of 
both layers due to the factor $\gamma_{i}$. In turn, these matrix elements depend on $p_{i}$ then cannot be dropped. By replacing $W_{\alpha}^{(i)}$ in the first term of eq.(12) an effective independent Hamiltonian for both graphene layers is found where the energy eigenvalues of $H_{0 S}+H_{e f}^{\prime}$ reads

$$
\epsilon_{s_{1}, p_{1} ; s_{2}, p_{2}}^{\prime}=\frac{g^{2}}{2 \hbar \omega} \sum_{i=1}^{2} \gamma_{i}^{2} \Delta_{i}\left(p_{i}\right)+\sum_{i=1}^{2} s_{i} v_{F} \eta_{i}\left(p_{i}\right) p_{i}
$$

where $s_{i}= \pm 1$ for both layers and indicates the valence $(-)$ and conduction $(+)$ bands respectively, $p_{i}$ is the wave-vector norm of electrons in each layer and

$$
\eta_{i}\left(p_{i}\right)=\sqrt{\left(1-\frac{g^{2} \gamma_{i}^{2}}{2\left(\hbar^{2} \omega^{2}-4 v_{F}^{2} p_{i}^{2}\right)}\right)\left(1+\frac{g^{2} \gamma_{i}^{2}}{2\left(\hbar^{2} \omega^{2}-4 v_{F}^{2} p_{i}^{2}\right)}\right)}
$$

and we have called $\Delta_{i}\left(p_{i}\right)=\frac{2 v_{F}^{2} p_{i}^{2}-\hbar^{2} \omega^{2}}{\hbar^{2} \omega^{2}-4 v_{F}^{2} p_{i}^{2}}$. The Fermi velocity correction at order $g^{2}$ in each layer can be obtained by computing $v_{F}^{\prime(i)}\left(p_{i}\right)=\frac{\partial \epsilon_{s_{1}, p_{1} ; s_{2}, p_{2}}}{\partial p_{i}}$ and is different in each layer due to the factor $\gamma_{i}$ and tends to $v_{F}$ for both layers when $g \rightarrow 0$ as it is shown in figure 2a).

Near the Dirac point in both layers, the effective Fermi velocity is $v_{F}^{\prime(i)} \sim v_{F} \sqrt{1-\frac{g^{4} \gamma_{i}^{4}}{4 \hbar^{4} \omega_{m}^{4}}}-\frac{2 v_{F}^{2} \gamma_{i}^{2} g^{2}}{\hbar^{3} \omega^{3}} p$ which is smaller than the bare Fermi velocity in each layer. This behavior can be experimentally detected for the condition $k_{F}\left|d_{2}-d_{1}\right|>1$, that is fulfilled for negligible $p$. The opposite condition $k_{F}\left|d_{2}-d 1\right|<1$ implies that $d<\left\langle r_{0}\right\rangle$ which is favourable for the hole-electron pairing for small interlayer separation $\left|d_{2}-d_{1}\right|$ as it is studied in [44]. The dispersion relation given by eq.(15), considering both layers with identical small carrier density $n$, then $k_{1}=k_{2}=k_{F}=\sqrt{\pi n}$ is plotted as a function of $\epsilon=v_{F} p$ in figure $2 \mathrm{~b}$ ). In dashed lines the asymptotic pristine double-layer graphene dispersion relation is shown $\epsilon_{ \pm}= \pm 2 v_{F} p$ (the degenerate band $\epsilon_{0}=0$ is not shown). Near the resonance point $p_{\text {res }}=\frac{\hbar \omega}{2 v_{F}}$, the dispersion relation shows a dip which is the precursor of a bandgap formation in the system. This forbidden region for $p_{F}$ is a novel feature of double-layer graphene that does not appear in normal 2D systems. The square root renormalization (eq.(16)) of the pristine graphene dispersion relation implies a forbidden region in the momentum $p_{F}$ when any of the factors inside the square root of eq.(16) is negative. The forbidden region in energy reads

$$
\Delta \epsilon_{i}^{\text {forbidden }}=\frac{1}{2} \sqrt{\hbar^{2} \omega^{2}+\frac{g^{2} \gamma_{i}^{2}}{2}}-\frac{1}{2} \sqrt{\hbar^{2} \omega^{2}-\frac{g^{2} \gamma_{i}^{2}}{2}} \sim \frac{\gamma_{i}^{2} g^{2}}{4 \hbar \omega}+O\left(g^{4}\right)
$$

This forbidden region implies a complex value of $\epsilon^{\prime}$ in eq.(15) and this implies that these energy states are broadened (see figure 2b)). The scattering time is given by $\tau=1 / 2 \operatorname{Im} \epsilon^{\prime}$ and vanishes at $p=\frac{\hbar \omega}{2 v_{F}}$ which implies that the broadening is larger near the edge of the forbidden region $\Delta \epsilon_{i}^{\text {forbidden }}$ and vanishes at the middle (see figure 2b). In turn, by using that $g=e \sqrt{\frac{2 \pi \hbar v_{F}^{2}}{\epsilon \omega_{m} V}}$ and that the energy stored in a capacitor $C=\frac{\epsilon S}{d}$ of parallel plates is $W=\frac{1}{2} \frac{Q^{2}}{C}$, where $S$ is the area of the plate, $d$ is the distance between them and $\epsilon$ the dielectric constant, the forbidden region $\frac{\gamma_{i} g^{2}}{4 \hbar \omega}$ can be written as the energy stored in a capacitor given by the respective layer at distance $d_{i}$ to the mirror. That is, by putting $\frac{\gamma_{i} g^{2}}{4 \hbar \omega}=W$, then $C_{i}=\frac{\epsilon S}{d_{i}^{e f}}$, where in this case $S$ is the area of the layers and $d_{i}^{e f}=\frac{\gamma_{i}^{2} \pi v_{F}^{2}}{\omega^{2} L}=\frac{\gamma_{i}^{2}}{m^{2} \pi}\left(\frac{v_{F}}{c}\right)^{2} L$ is the effective distance of the capacitor, where we have replaced $\omega=\frac{c m \pi}{L}$ where $m$ is the mode index. For large modes, higher photon frequencies and small effective distances are found. In the case we consider that $\frac{d_{i}}{L}<<1$, where $d_{i}$ is the distance of the $i$-layer of graphene to one of the mirrors, then $\gamma_{i} \sim \frac{m \pi d_{i}}{L}$ and $d_{i}^{e f}=\left(\frac{v_{F}}{c}\right)^{2} \frac{d_{i}^{2}}{L}$.

In order to study the effective Hamiltonian $H_{e f}^{\prime \prime}$ that contains the coupling between layers we can

5
7
8
9
6


a)

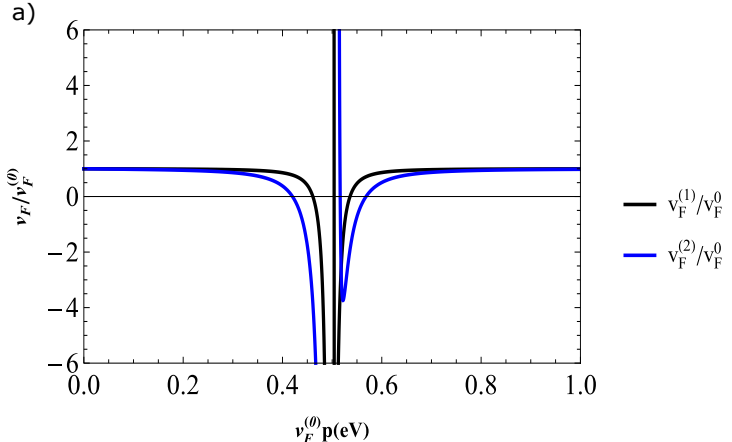

b)

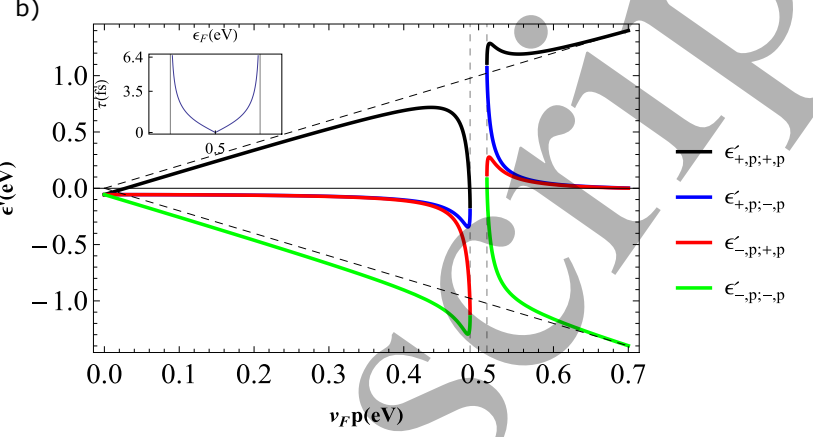

Figure 2: a) Fermi velocity renormalization for both layers where $g=0.5 \mathrm{eV}, \hbar \omega=1 \mathrm{eV}, \gamma_{1}=0.3$ and $\gamma_{2}=0.6$. b) Dispersion relation $\epsilon^{\prime}$ for identical Fermi momentum $p_{1}=p_{2}=p$ and where $g=0.5 \mathrm{eV}$, $\hbar \omega=1 \mathrm{eV}, \gamma_{1}=0.3$ and $\gamma_{2}=0.6$. Inset figure: time relaxation as a function of energy in the gap zone. Dashed lines indicate pristine dispersion relation $2 v_{F} p$ and $-2 v_{F} p$.

replace $W_{\alpha}^{(i)}$ and $X_{\alpha \alpha^{\prime}}^{(i)}$ in eq.(13), and by restoring the Pauli matrices $\sigma_{x}$ and $\sigma_{y}$ we obtain

$$
\begin{gathered}
H_{e f}^{\prime \prime}=\gamma_{1} \gamma_{2} R\left(p_{1}\right)\left[\cos \left(\frac{\theta}{2}+\varphi_{1}\right) \sigma_{x}^{(2)}+\sin \left(\frac{\theta}{2}+\varphi_{1}\right) \sigma_{y}^{(2)}\right] \sigma_{z}^{(1)}+ \\
\gamma_{1} \gamma_{2} R\left(p_{2}\right)\left[\cos \left(\frac{\theta}{2}-\varphi_{2}\right) \sigma_{x}^{(1)}-\sin \left(\frac{\theta}{2}-\varphi_{2}\right) \sigma_{y}^{(1)}\right] \sigma_{z}^{(2)}+ \\
\frac{\gamma_{1} \gamma_{2}}{\hbar \omega} r_{2}^{C}\left(p_{1}, p_{2}\right)\left(\sigma_{x}^{(1)} \sigma_{x}^{(2)}-\sigma_{y}^{(1)} \sigma_{y}^{(2)}\right)+\frac{\gamma_{1} \gamma_{2}}{\hbar \omega} r_{2}^{S}\left(p_{1}, p_{2}\right)\left(\sigma_{x}^{(1)} \sigma_{y}^{(2)}+\sigma_{y}^{(1)} \sigma_{x}^{(2)}\right)+ \\
\frac{\gamma_{1} \gamma_{2}}{2 \hbar \omega} r\left(p_{1}, p_{2}, \omega\right)\left[\cos \theta \sigma_{\mathbf{1}} \cdot \sigma_{\mathbf{2}}+\sin \theta\left(\sigma_{\mathbf{1}} \times \sigma_{\mathbf{2}}\right) \cdot \widehat{e}_{z}\right]
\end{gathered}
$$

where $R\left(p_{i}\right)=\frac{v_{F} p_{i}}{\hbar^{2} \omega^{2}-4 v_{F}^{2} p_{i}^{2}}$ multiplies trigonometric functions that couple the twist angle $\theta$ with the angle in the momentum space of each layer. The coefficients $r_{2}^{C}$ and $r_{2}^{S}$ reads

and $r$ reads

$$
\begin{aligned}
& r_{2}^{C}\left(\mathbf{p}_{1}, \mathbf{p}_{2}\right)=\sum_{i=1,2} \frac{v_{F}^{2} p_{i}^{2} \cos \left(2 \varphi_{i}\right)}{\hbar^{2} \omega^{2}-4 v_{F}^{2} p_{i}^{2}} \\
& r_{2}^{S}\left(\mathbf{p}_{1}, \mathbf{p}_{2}\right)=\sum_{i=1,2} \frac{v_{F}^{2} p_{i}^{2} \sin \left(2 \varphi_{i}\right)}{\hbar^{2} \omega^{2}-4 v_{F}^{2} p_{i}^{2}}
\end{aligned}
$$

$$
r\left(p_{1}, p_{2}, \omega\right)=\sum_{i=1,2} \Delta_{i}\left(p_{i}\right)
$$

The most interesting term in eq.(18) is the last one because it does not vanish at the Dirac point and where $\sigma_{1} \cdot \sigma_{2}$ is the dot product of Pauli matrices and $\left(\sigma_{1} \times \sigma_{2}\right) \cdot \widehat{e}_{z}$ is the $z$-component of the vector product.

We can apply second-order perturbation theory with the transformed Hamiltonian of eq.(6) considering $[S, V]$ the perturbation. The eigenvectors of the unperturbed $H_{0 S}+H_{e f}^{\prime}$ can be written as

$$
\left|\Psi_{p_{1}, s_{1} ; p_{2}, s_{2}}\right\rangle=\frac{e^{i \mathbf{p}_{1} \cdot \mathbf{r}_{1}}}{\sqrt{2 S}} \frac{e^{i \mathbf{p}_{2} \cdot \mathbf{r}_{2}}}{\sqrt{2 S}}\left(\left|A_{1}\right\rangle+s_{1} e^{i \varphi_{p_{1}}}\left|B_{1}\right\rangle\right) \otimes\left(\left|A_{2}\right\rangle+s_{2} e^{i \varphi_{p_{2}}}\left|B_{2}\right\rangle\right)
$$

which is a tensor product of the respective eigenvectors of the two graphene layers. The second order perturbation of the energy is given by

$$
\epsilon_{p_{1}, s_{1} ; p_{2}, s_{2}}^{\prime \prime}=\sum_{i=1}^{2} s_{i} v_{F} p_{i}+\frac{g^{2}}{2}\left\langle\Psi_{p_{1}, s_{1} ; p_{2}, s_{2}}\left|H_{e f}^{\prime}+H_{e f}^{\prime \prime}\right| \Psi_{p_{1}, s_{1} ; p_{2}, s_{2}}\right\rangle
$$



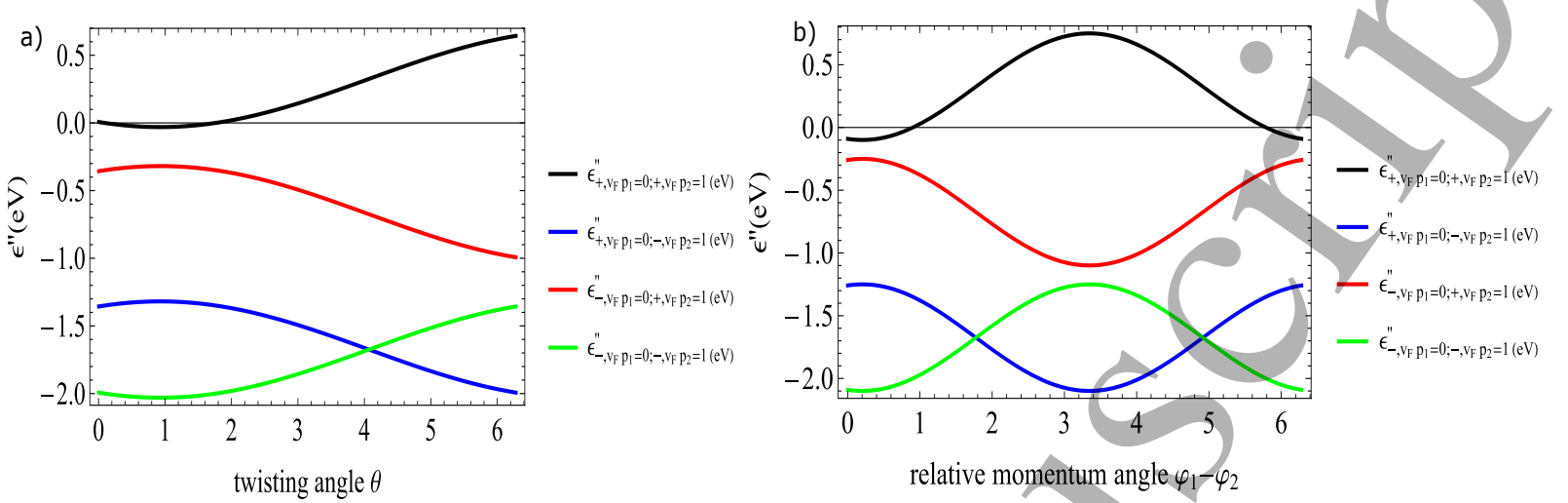

Figure 3: a) Dispersion relation $\epsilon^{\prime \prime}$ as a function of the twisting angle $\theta$ with $\epsilon_{1}=v_{F} p_{F}=0, \epsilon_{2}=$ $v_{F} p_{2}=1 \mathrm{eV}, g=0.5 \mathrm{eV}, \hbar \omega=0.05 \mathrm{eV}, \gamma_{1}=0.3$ and $\gamma_{2}=0.6$ and $\varphi_{1}-\varphi_{2}=\pi / 4$. b) Dispersion relation $\epsilon^{\prime \prime}$ as a function of the momentum angles $\varphi_{1}-\varphi_{2}$ with $\epsilon_{1}=v_{F} p_{F}=0, \epsilon_{2}=v_{F} p_{2}=1 \mathrm{eV}, g=0.5 \mathrm{eV}$, $\hbar \omega=0.05 \mathrm{eV}, \gamma_{1}=0.3$ and $\gamma_{2}=0.6$ and $\theta=\pi / 4$.

The eigenvectors of $H_{e f}^{\prime}$ are identical to those of $\left\langle H_{0 S}\right.$, then the term $\frac{g^{2}}{2}\left\langle\Psi_{p_{1}, s_{1} ; p_{2}, s_{2}}\left|H_{e f}^{\prime}\right| \Psi_{p_{1}, s_{1} ; p_{2}, s_{2}}\right\rangle$ introduces the eigenvalues of eq.(15). The correction due to $H_{e f}^{\prime \prime}$ reads

$$
\frac{g^{2}}{2}\left\langle\Psi_{p_{1}, s_{1} ; p_{2}, s_{2}}\left|H_{e f}^{\prime \prime}\right| \Psi_{p_{1}, s_{1} ; p_{2}, s_{2}}\right\rangle=-\frac{g^{2} \gamma_{1} \gamma_{2}}{2 \hbar \omega} s_{1} s_{2}\left[\cos \left(\varphi_{1}-\varphi_{2}\right) \cos \left(\frac{\theta}{2}\right)+\xi\left(p_{1}, p_{2}\right) \sin \left(\varphi_{1}-\varphi_{2}\right) \sin \left(\frac{\theta}{2}\right)\right]
$$

where

$$
\xi\left(p_{1}, p_{2}\right)=\frac{2 \hbar^{2} \omega^{2}\left(v_{F}^{2} p_{1}^{2}-v_{F}^{2} p_{2}^{2}\right)}{\left(\hbar^{2} \omega^{2}-4 v_{F}^{2} p_{1}^{2}\right)\left(\hbar^{2} \omega^{2}-4 v_{F}^{2} p_{2}^{2}\right)}
$$

where we have replaced $\theta_{1}=-\theta_{2}=\frac{\theta}{2}$. The, correction at second order in $g$ given by last equation implies that when both electrons or holes in each layer have identical Fermi momentum $p_{1}=p_{2}=p_{F}$, then

$$
\left\langle\Psi_{p_{1}, s_{1} ; p_{2}, s_{2}}\left|H_{e f}^{\prime \prime}\right| \Psi_{p_{1}, s_{1} ; p_{2}, s_{2}}\right\rangle_{p_{1}=p_{2}}=-\frac{g^{2} \gamma_{1} \gamma_{2}}{2 \hbar \omega} s_{1} s_{2} \cos \left(\varphi_{1}-\varphi_{2}\right) \cos \left(\frac{\theta}{2}\right)
$$

then the correction decreases the energy when both layers are electron doped $\left(s_{1}=s_{2}=1\right)$ or hole doped $\left(s_{1}=s_{2}=-1\right)$ whenever $\theta \neq \pi$ or $\varphi_{1}-\varphi_{2} \neq \frac{\pi}{2}$ which is in concordance with a superfluid phase of interlayer excitons $([45],[46]$ and $[47])$. When the twist angle vanishes, the second order correction reads

$$
\left\langle\Psi_{p_{1}, s_{1} ; p_{2}, s_{2}}\left|H_{e f}^{\prime \prime}\right| \Psi_{p_{1}, s_{1} ; p_{2}, s_{2}}\right\rangle_{\theta=0}=-\frac{g^{2} \gamma_{1} \gamma_{2}}{2 \hbar \omega} s_{1} s_{2} \cos \left(\varphi_{1}-\varphi_{2}\right)
$$

Last equation implies that when the Fermi level $\mu_{i}$ is such that $\mu_{1}, \mu_{2}>0$ both layers are electron doped, then $s_{1} s_{2}=1$ and the minimum of $\epsilon_{+, p_{1} ;+, p_{2}}^{\prime \prime}$ is obtained for $\varphi_{1}=\varphi_{2}$. Identically, when $\mu_{1}<0$ and $\mu_{2}<0, \epsilon_{-, p_{1} ;-, p_{2}}^{\prime \prime}$ minimizes when $\theta_{1}=\theta_{2}$ for both holes doped layers. For $\mu_{1}>0$ and $\mu_{2}<0$ and $\mu_{1}<0$ and $\mu_{2}>0$, the minimum $\epsilon_{+, p_{1} ;-, p_{2}}^{\prime \prime}$ and $\epsilon_{-, p_{1} ;+, p_{2}}^{\prime \prime}$ is obtained for $\theta_{2}=\theta_{1}+\pi$. For electron-hole doped layers, the angle-correlation induces a coherence given by a net flow of current between layers [48]. On the other side, when $p_{1} \neq p_{2}$ in eq.(23) and $\theta=\pi$, the correction to the energy due to $H_{e f}^{\prime \prime}$ is modulated by $\sin \left(\varphi_{1}-\varphi_{2}\right)$. These results indicate a momentum angle-twisting angle correlation that induce a coherence between electrons in different layers (see figure 3b)). In turn, it can be seen that for specific rotation and relative momentum angle, two bandgaps appear, which exhibits novel insulating phases modulated by the relative direction of propagation of electrons in each layer. When both graphene layers are neutral, the electrons are located at the Dirac point, $p_{1}=p_{2}=0$ and we can 
Figure 4: Dispersion relation $\epsilon_{ \pm n}^{(i)}$ of double-layer graphene at the Dirac point where $g=0.5 \mathrm{eV}, \hbar \omega=$ $1 \mathrm{eV}, \gamma_{1}=0.3$ and $\gamma_{2}=0.6$.

exploit eq.(11) to find the Hamiltonian $H_{D P}$ at order $g^{2}$

$$
\begin{gathered}
H_{D P}=\left[1-\frac{g^{2}}{\hbar^{2} \omega^{2}}\left(\gamma_{1}^{2} \sigma_{z}^{(1)}+\gamma_{2}^{2} \sigma_{z}^{(2)}\right)\right] \hbar \omega a^{\dagger} a-\frac{g^{2}}{2 \hbar \omega}\left(\gamma_{1}^{2} \sigma_{z}^{(1)}+\gamma_{2}^{2} \sigma_{z}^{(2)}\right) \\
-J_{D P}\left[\cos \theta \sigma_{\mathbf{1}} \cdot \sigma_{\mathbf{2}}+\sin \theta\left(\sigma_{1} \times \sigma_{\mathbf{2}}\right) \cdot \widehat{e}_{z}\right]
\end{gathered}
$$

where a constant term $-\frac{g^{2}}{2}\left(\frac{\gamma_{1}^{2}}{\hbar \omega}+\frac{\gamma_{2}^{2}}{\hbar \omega}\right) I$ has been dropped and $J_{D P}=\frac{g^{2} \gamma_{1} \gamma_{2}}{2 \hbar \omega}$ is the pseudospin exchange coupling between layers. The minus sign indicates a pseudo-ferromagnetic coupling, where the sublattice basis are anti-correlated. When $\theta \equiv 0$, the effective interaction between layers reduces to $-J_{D P} \sigma_{\mathbf{1}} \cdot \sigma_{\mathbf{2}}$ and for orthogonal twist $\theta=\pi / 2$, the effective interaction reduces to $-J_{D P}\left(\sigma_{\mathbf{1}} \times \sigma_{\mathbf{2}}\right) \cdot \widehat{e}_{z}$. The effective interaction can be very small compared to the free evolution due to $H_{0}$. However, by moving to the rotating frame, $H_{0}$ no longer appears and the interaction becomes important. On the other side, by tuning $\omega$ with the cavity height $L$ and the refractive index, $J_{D P}$ can be large and the pseudo-spin interaction between layers is predominant in the Hamiltonian of eq.(27). Last result is similar for two Cooper pair box qubits coupled to a transmission line resonator [49]. In this frame, the two-qubit interaction becomes important and can be used to entangle the two qubits together and perform gates on them. In turn, the entanglement can be tuned through $\omega, \gamma_{1}$ and $\gamma_{2}$. The effective distance of the capacitor between layers is $d_{e f}^{G-G}=2 \pi\left(\frac{v_{F}}{c}\right)^{2} \frac{d_{1} d_{2}}{L}$ and can be compared with the quantum capacitance of double-layer graphene [50]. From Hamiltonian $H_{D P}$ it can be seen that the A. C. Stark effect split the photon energy state $\hbar \omega n$ in $\hbar \omega \pm \frac{g^{2}}{\hbar \omega}\left(\gamma_{1}^{2} \pm \gamma_{2}^{2}\right)$. More insight into the dispersion relation of double-layer graphene inside the microcavity at the Dirac point for weak coupling is gained by obtaining the full set of eigenvalues of $H_{D P}$, where wê can consider the basis $\{|A, A, n\rangle,|A, B, n\rangle,|B, A, n\rangle,|B, B, n\rangle\}$ and the energy of the dressed states reads

$$
\begin{gathered}
\epsilon_{n, \pm}^{(1)}=\hbar \omega n \pm \frac{g^{2}}{2 \hbar \omega}(2 n+1)\left(\gamma_{1}^{2}+\gamma_{2}^{2}\right) \\
\epsilon_{n, \pm}^{(2)}=\hbar \omega n \pm \frac{g^{2}}{2 \hbar \omega} \sqrt{\left(\gamma_{1}^{4}+\gamma_{2}^{4}\right)(2 n+1)-2 \gamma_{1}^{2} \gamma_{2}^{2}(4 n(n+1)-1)}
\end{gathered}
$$

From figure (4) it can be seen that the spectrum contains a set (different $n$ ) of four non-degenerated energy eigenvalues as a function of of the cavity photon frequency. In turn, it can be seen that these eigenvalues naturally separates into two branches (valence and conduction bands) and the splitting between states depends on $n$. The cavity resonance $\omega_{\text {res }}$ is located at $\omega_{\text {res }}=0$ because both layers are neutral. 
Current technology allows the experimental realization of few-layer graphene in large areas by both mechanical exfoliation or chemical techniques with controlled stacking order ([51] and [52]). The schematic setup of our proposed system is depicted in figure 1, where the two parallel graphene sheets can be suspended or on top of a substrate. The layer separation must be $>3 \AA$ in order to prevent tunneling between the graphene sheets. An operational procedure to detect the non-local correlation in double-layer graphene involves applying an external voltage on both layers, which can vary the carrier concentration in the material [53]. An usual procedure to change the charge concentration is to use graphene as the second parallel plate of a capacitor, where the first plate $\mathrm{is}_{\mathrm{SiO}_{2}}$ and a backgate voltage is applied perpendicular to the graphene sheet which creates an electrostatic potential drop between the sample and the gate electrode and shifts the Fermi level [54]. The distance between graphene layers should be an order of magnitude larger than the capacitor in order to not change the boundary conditions for the electromagnetic field used in the calculations [55]. An improvement to the setup is to introduce a dielectric in the whole cavity that changes the refractive index and the velocity of light in order to decrease the time intervals at which the back-gate voltages are switched on and off in order to detect the entanglement at early times [56]. In turn, the two sheets are separated in order to prevent tunneling between the sheets and electron-hole recombination in the case one of the sheet is hole doped and the Coulomb drag can be neglected [13]. As well there can be two separate electrical contacts to the two layers, allowing independent control over the carrier density in each sheet. For negligible carrier density, the effective Hamiltonian is given by eq.(27) and by allowing the electromagnetic wave inside the microcavity to be turned on and off with a duration $T$, the time evolution of the density operator of both layers can be computed. Considering an initial state in which both electrons are in an eigenstate of the free Hamiltonian or in a particular sublattice basis, the time-evolved density operator is $\rho_{T}=U \rho_{0} U^{\dagger}$, where $U$ is the evolution operator expanded in $g$. Quantum correlations between layers appears as non-diagonal elements of the density matrix in the basis chosen, for example $X$ states [57]. These nondiagonal elements will depends strongly in the switching function, $d_{1}, d_{2}, L$ and $T$. The generation of entanglement between graphene layers through the electromagnetic field in the microcavity allows us to makes these materials attractive to be applied to different branches of quantum optics, such as quantum information [58], polaritonics [59], quantum teleportation ([60] and [61], quantum cryptography ([62] and [63]) and creates a basis of modern technological applications ([64], [65]). A simple inspection of the effective Hamiltonian $H_{L-L}=-J_{D P}\left[\cos \theta \sigma_{1} \cdot \sigma_{2}+\sin \theta\left(\sigma_{1} \times \sigma_{\mathbf{2}}\right) \cdot \widehat{e}_{z}\right]$ implies that an initial state $|\Psi(0)\rangle=\left|A_{1}, B_{2}\right\rangle$ evolves to $e^{-i H_{L-L t}}|\Psi(0)\rangle=\cos \left(J_{D P} t\right)\left|A_{1}, B_{2}\right\rangle-i e^{-i \theta} \sin \left(J_{D P} t\right)\left|B_{1}, A_{1}\right\rangle$, then the twisting angle $\theta$ can be used to tune the entanglement between the layers.

\section{Conclusions}

In summary, we have presented a theoretical procedure, based on the Schrieffer-Wolf transformation of the Hamiltonian, to obtain the effective interaction between electrons in different rotated layers of graphene inside a planar microcavity. We have shown that considering a monochromatic clockwise polarized electromagnetic wave that interacts with each layer through the minimal coupling, both layers gets coupled through the interchange of a second order transition that involves a virtual photon emmited from one layer and absorbed in the other layer. The effective interaction depends strongly in the twisting angle $\theta$ of the layers, the cavity photon frequency $\omega$ and the distances between the layers and the mirrors of the cavity. It was shown that the Fermi velocity in each layer is different and a resonance appears. In turn, a band gap appears around the resonance due to imaginary values of the energy obtained. It was shown that the dispersion relation decreases when the momentum angle of the electrons are $\varphi_{1}=\varphi_{2}$ when the Fermi level is above or below the Dirac point for both layers and decreases when $\varphi_{2}=\varphi_{1}+\pi$ when both layers are identically electron-hole doped. These results implies an anglecorrelation coherence that can induce a net flow of current between the layers with a twisting angle $\theta$ modulation. In turn, when both layers are not doped identically and $\theta=\pi / 2$, the energy dispersion decreases when $\varphi_{2}=\varphi_{1}$. This unexpected behavior for different $\theta$ is the precursor of a momentum angletwisting angle correlation between layers which indicates that the transport properties of the system 
are highly sensitive to $\theta$. Finally, the dispersion relation was found at the Dirac point, showing that the effective interaction can be written compactly as $-J_{D P}\left[\cos \theta \sigma_{\mathbf{1}} \cdot \sigma_{\mathbf{2}}+\sin \theta\left(\sigma_{\mathbf{1}} \times \sigma_{\mathbf{2}}\right) \cdot \widehat{e}_{z}\right]$, where a pseudo-ferromagnetic coupling $J_{D P}$ appears between layers and can be used to entangle two qubits given by the sublattice basis in each layer and perform gates on them through the manipulation of the twisting angle $\theta$.

\section{Acknowledgment}

This paper was partially supported by grants of CONICET (Argentina National Research Council) and Universidad Nacional del Sur (UNS) and by ANPCyT through PICT 1770, and PIP-CONICET Nos. 114-200901-00272 and 114-200901-00068 research grants. J. S. A. is member of CONICET.

\section{References}

[1] A. Geim, Science 324, 1530 (2009).

[2] S. Das Sarma, S. Adam, E. H. Hwang, and E. Rossi, Rev. Mod. Phys. 83, 407 (2011).

[3] A. H. Castro Neto, F. Guinea, N. M. R. Peres, K. S. Novoselov, and A. K. Geim, Rev. Mod. Phys. 81, 109 (2009).

[4] J. I. A. Li, Q. Shi, Y. Zeng, K. Watanabe, T. Taniguchi, J. Hone and C. R. Dean, Nature Physics, 15, 898-903 (2019).

[5] M.Y. Kharitonov and K. B. Efetov, Phys. Rev. B, 78, 241401 (2008).

[6] I. Sodemann, D. A. Pesin, A. H. MacDonald, Phys. Rev. B 85, 195136 (2012).

[7] M. Zarenia, A. Perali, D. Neillson and F. M. Peeters, Scientific Reports, 4 : 7319 (2014).

[8] R. E. V. Profumo, M. Polini, R. Asgari, R. Fazio and A. H. MacDonald, Phys. Rev. B, 82, 085443 (2010).

[9] E. Gibney, Nature 565, 15-18 (2019).

[10] M. S. Scheurer, Naturê 572 (7767): 40-41 (2019).

[11] Y. Cao, V. Fatemi, S. Fang, S. et al. Nature 556, 43-50 (2018).

[12] T Stauber and G. Gomez-Santos, New J. Phys. 14, 105018 (2012).

[13] R. V. Gorbachev, A. K. Geim, M. I. Katsnelson, K. S. Novoselov, T. Tudorovskiy, I. V. Grigorieva, A. H. MacDonald, S. V. Morozov, K. Watanabe, T. Taniguchi and L. A. Ponomarenko, Nature Physics 8, 896-901 (2012).

[14] J. S. Ardenghi, Phys. Rev. D, 98, 045006 (2018).

[15] O. V. Kibis, Phys. Rev. B 81, 165433 (2010).

[16] E. T. Jaynes and F. W. Cummings, Proc. IEEE, 51(1), 89-109 (1963).

[17] C. Cohen-Tannoudji, J. Dupont-Roc and G. Grynberg Atom-Photon Interactions: Basic Processes and Applications (Chichester, Wiley, 1988).

[18] M. O. Scully and M. S. Zubairy, Quantum Optics (Cambridge, University Press, 2001). 
[19] J. Kasprzak, M. Richard, S. Kundermann, A. Baas, P. Jeambrun, J. M. J. Keeling, F. M,Marchetti, M. H. Szymanska, R. Andr'e, J. L. Staehli, V. Savona, P. B. Littlewood, B. Deveaud and L. S. Dang, Nature 443, 409 (2006).

[20] H. Deng, H. Haug, and Y. Yamamoto, Rev. Mod. Phys. 82, 1489 (2010).

[21] J. P. Reithmaier, G. Sek, A. Loffler, C. Hofmann, S. Kuhn, S. Reitzenstein, L. V. Keldysh, V. D. Kulakovskii, T. L. Reinecker and A. Forhel, Nature 432, 197 (2004).

[22] T. Yoshie, A. Scherer, J. Heindrickson, G. Khitrova, H. M. Gibbs, G. Rupper, C. Ell, O. B. Shchekin and P. G. Deppe, Nature 432, 200 (2004).

[23] T. C. H. Liew, I. A. Shelykh, G. Malpuech, Physica E 43, 1543 (2011).

[24] S. Christopoulos, G. Baldassarri H“oger von H“ogersthal, A. Grundy, P. G. Lagoudakis, A. V. Kavokin, J. J. Baumberg, G. Christmann, R. Butt'e, E. Feltin, J.-F. Carlin, and N. Grandjean, Phys. Rev. Lett. 98, 126405 (2007).

[25] C. Schneider, A. Rahimi-Iman, N. Y. Kim, J. Fischer, I. G. Savenko, M. Amthor, M. Lermer, A. Wolf, L. Worschech,V. D. Kulakovskii, I. A. Shelykh, M. Kamp, S. Reitzenstein, A. Forchel, Y. Yamamoto, and S. H*ofling, Nature 497, 348 (2013).

[26] T. K. Paraiso, M. Wouters,Y. Leger, F. Morier-Genoud, B. Deveaud-Pledran, Nature Mater. 9, 655 (2010).

[27] C. Anton, T. C. H. Liew, J. Cuadra, M. D. Martin, P. S. Eldridge, Z. Hatzopoulos, G. Stavrinidis, P. G. Savvidis, and L. Vina, Phys. Rev. B 88, 245307 (2013).

[28] R. Johne, N. A Gippius, G. Pavlovic, D. D. Solnyshkov, I. A. Shelykh, and G. Malpuech, Phys. Rev. Lett. 100, 240404 (2008)

[29] F.J. Lopez-Rodriguez and G.G. Naumis, Phys. Rev. B 78, 201406(R) (2008).

[30] T. Oka and H. Aoki, Phys. Rev. B 79, 081406(R) (2009).

[31] O. V. Kibis, O. Kyriienko and I. A. Shelykh, Phys. Rev. B 84, 195413 (2011).

[32] S. E. Savelev and A. S. Alexandrov, Phys. Rev. B 84, 035428 (2011).

[33] H. L. Calvo, H. M. Pastawski, S. Roche and L. E. F. Foa Torres, Appl. Phys. Lett. 98, 232103 (2011).

[34] Y. Zhou and M.W. Wu, Phys. Rev. B 83, 245436 (2011).

[35] S. V. Syzranov, M. V. Fistul and K.B. Efetov, Phys. Rev. B 78, 045407 (2008).

[36] A. Iurov, G. Gumbs, O. Roslyak and D. Huang, J. Phys.: Condens. Matter 25, 135502 (2013).

[37] G. Usaj, P.M. Perez-Piskunow, L. E. F. Foa Torres and C. A. Balseiro, Phys. Rev. B 90, 115423 (2014).

[38] L. E. F. Foa Torres, P. M. Perez-Piskunow, C. A. Balseiro and G. Usaj, Phys. Rev. Lett. 113, $266801(2014)$.

[39] S. Bravyi, D. P. DiVincenzo and D. Loss, Ann. Phys., 326, 2793 (2011).

[40] J.R.Schrieffer and P.A.Wolff, Phys. Rev. 149, 2 (1966).

[41] C. Cohen-Tannoudji, J. Dupont-Roc, and G. Grynberg, Photons and Atoms: Introduction to Quantum Electrodynamics (Wiley-VCH, 1989). 
[42] R. Bistritzer and A. H. MacDonald, Proc. Natl. Acad. Sci., 108, 30 12233-12237 (2011).

[43] P. O. Löwdin, J. Mol. Spectrosc., 10: 12-33 (1963).

[44] A. Perali, D. Neilson, and A. R. Hamilton, Phys. Rev. Lett., 110, 146803 (2013).

[45] Eisenstein, J. P. Exciton condensation in bilayer quantum Hall systems. Annu. Rev./Condens. Matter Phys. 5, 159-181 (2014).

[46] Li, J. I. A., Taniguchi, T., Watanabe, K., Hone, J. \& Dean, C. R. Nat. Phys. 13, 751-755 (2017).

[47] Liu, X., Taniguchi, T., Watanabe, K., Halperin, B. \& Kim, P. Nat. Phys. 13, 746-750 (2017).

[48] M. P. Mink, H. T. C. Stoof and R.A. Duine, Phys. Rev. B, 84, 155409 (2011).

[49] A. Blais, R.-S. Huang, A. Wallraff, S. M. Girvin, R. J. Schoelkopf, Phys. Rev. A 69, 062320 (2004).

[50] F. Parhizgar, A. Qaiumzadeh and R.Asgari, Phys. Rev. B, 96, 075447 (2017).

[51] C. Berger, Z. Song, T. L. Xuebin Li, A. Y. Ogbazghi, R. Feng, Z. Dai, A. N. Marchenkov, E. H. Conrad, P. N. First and W. A. de Heer, J. Phys. Chem. B 108,19912 (2004).

[52] C.-J. Shih, A. Vijayaraghavan, R. Krishnan, R. Sharma, J.-H. Han, M.-H. Ham, Z. Jin, S. Lin, G. L.C. Paulus, N. F. Reuel, Q. H. Wang, D. Blankschtein and M. S. Strano, Nat. Nanotechnol. 6, 439-445 (2011).

[53] M. F. Craciun, S. Russo, M. Yamamoto, and S. Tarucha, Nano Today 6, 42 (2011).

[54] A. Das, B. Chakraborty, and K. Sood, Graphene and Its Fascinating Attributes (World Scientific, Singapore, 2011), Chap. 7.

[55] S. M. Badalyan and F. M. Peeters, Phys. Rev. B 85, 195444 (2012).

[56] K. Tsukagoshi, H. Miyazaki, S.-L. Li, A. Kumatani, H. Hiura, and A. Kanda, Graphene and its Fascinating Attributes (World Scientific, Singapore, 2011), p. 179.

[57] T. Yu and J. H. Eberly, Quant. Inf. Comp. 7, 459 (2007).

[58] M. A. Nielsen and I. Chuang, Quantum Computation and Quantum Information (Cambridge University Press, Cambridge, 2000).

[59] A. V. Kavokin, J. J. Baumberg, G. Malpuech and F. P. Laussy, Microcavities (Oxford University Press, Oxford, 2007).

[60] C. H. Bennett, G. Brassard, C. Crepeau, R. Jozsa, A. Peres and W. K. Wootters, Phys. Rev. Lett. 70, 1895-1899 (1993).

[61] D. Bouwmeester, J. W. Pan, K. Mattle, M. Eibl, H. Weinfurter and A. Zeilinger, Nature 390, $575-579(1997)$.

[62] A. K. Ekert, Phys. Rev. Lett. 67, 661-663 (1991)

[63] N. Gisin, G. Ribordy, W. Tittel and H. Zbinden, Rev. Mod. Phys. 71, 145-195 (2002).

[64] J. Kasprzak, M. Richard, S. Kundermann, A. Baas, P. Jeambrun, J. M. J. Keeling, F. M. Marchetti, M. H. Szymanska, R. Andre, J. L. Staehli, V. Savona, P. B. Littlewood, B. Deveaud and L.S. Dang, Nature 443, 409-414 (2006).

[65] A. Baas, J. P. Karr, H. Eleuch and E. Giacobino, Phys. Rev. A 69, 023809 (2004). 